\title{
Transient Radio Signatures from Neutron Star Encounters with QCD Axion Miniclusters
}

\author{
Thomas D. P. Edwards $\odot,{ }^{1,2, *}$ Bradley J. Kavanagh $\odot,{ }^{3,2, \dagger}$ Luca Visinelli $\odot,{ }^{2,4, \hbar}$ and Christoph Weniger, ${ }^{2, \S}$ \\ ${ }^{1}$ The Oskar Klein Centre for Cosmoparticle Physics, AlbaNova University Center, \\ Roslagstullsbacken 21, SE-106 91 Stockholm, Sweden \\ ${ }^{2}$ Gravitation Astroparticle Physics Amsterdam (GRAPPA), Institute for Theoretical Physics Amsterdam \\ and Delta Institute for Theoretical Physics, University of Amsterdam, Science Park 904, 1098 XH Amsterdam, Netherlands \\ ${ }^{3}$ Instituto de Física de Cantabria (IFCA, UC-CSIC), Avenida de los Castros s/n, 39005 Santander, Spain \\ ${ }^{4}$ INFN, Laboratori Nazionali di Frascati, C.P. 13, 100044 Frascati, Italy
}

(Received 16 December 2020; revised 17 June 2021; accepted 16 July 2021; published 23 September 2021)

\begin{abstract}
The QCD axion is expected to form dense structures known as axion miniclusters if the Peccei-Quinn symmetry is broken after inflation. Miniclusters that have survived until today will interact with neutron stars (NSs) in the Milky Way to produce transient radio signals from axion-photon conversion in the NS magnetosphere. We quantify the properties of these encounters and find that they occur frequently $\left[\mathcal{O}(1-100)\right.$ day $\left.^{-1}\right]$, last between a day and a few months, are spatially clustered toward the Galactic Center, and can reach observable fluxes. These radio transients are within reach of current generation telescopes and therefore offer a promising pathway to discovering QCD axion dark matter.
\end{abstract}

DOI: 10.1103/PhysRevLett.127.131103

Introduction.-Peccei-Quinn (PQ) theory [1,2] predicts the existence of the QCD axion [3,4], which could simultaneously solve the strong- $C P$ problem and act as a compelling candidate for particle dark matter (DM) [5-8]. The QCD axion is the pseudo-Nambu-Goldstone boson [9-11] of the new global PQ symmetry. Laboratory searches are underway worldwide to directly detect this QCD axion [12-15]. Astrophysical observations are also a promising avenue for detecting the axion [16-20]. In particular, radio observations could be used to look for emission from the conversion of axions into photons in neutron star (NS) magnetospheres [21-27]. In this Letter, we propose and characterize a new class of radio source, arising from encounters between NSs and overdense structures known as axion miniclusters (AMCs) [28-33]. (We use the terms miniclusters and AMCs interchangeably.)

As axions fall toward a NS, they can resonantly convert into photons within the magnetosphere. This occurs at a radius $R_{c}$, at which the plasma frequency $\omega_{p}$ in the magnetosphere equals the axion mass $m_{a}$ [23]. Assuming a Goldreich-Julian model for the NS magnetosphere [34], the power radiated per unit solid angle is derived in the WentzelKramers-Brillouin and stationary phase approximations as [21-24]

Published by the American Physical Society under the terms of the Creative Commons Attribution 4.0 International license. Further distribution of this work must maintain attribution to the author(s) and the published article's title, journal citation, and DOI. Funded by SCOAP ${ }^{3}$.

$$
\frac{d \mathcal{P}_{a}}{d \Omega} \sim \frac{\pi}{3} g_{a \gamma \gamma}^{2} B_{0}^{2} \frac{R_{\mathrm{NS}}{ }^{6}}{R_{c}{ }^{3}} \frac{\rho_{a}}{m_{a}}
$$

where $\rho_{a}$ is the axion density at the conversion radius [23], $g_{\text {ary }}$ is the axion-photon coupling, and we have averaged over viewing angles. We also fix the rotation axis to be aligned with the NS dipole field and set the NS radius $R_{\mathrm{NS}}=10 \mathrm{~km}$ (see Supplemental Material for details of the NS modeling [35]). The power scales with $B_{0}^{2}$-where $B_{0}$ is the magnetic field strength at the NS poles - emphasizing why NSs are the most promising astrophysical target for these searches, with the highest known magnetic fields in the Universe $\mathcal{O}\left(10^{10}-10^{15}\right) \mathrm{G}$ [55]. The flux also scales with $\rho_{a}$, meaning that regions of large axion density - such as AMCs-can give rise to very bright radio sources.

AMCs are a generic feature of models in which the PQ symmetry is broken after the end of inflation [32]. Large spatial variations of the axion density around the QCD epoch lead to the formation of minicluster "seeds" [56], which collapse into gravitationally bound AMCs around matter-radiation equality [57]. This evolution has been confirmed by numerical simulations, which show that a significant fraction of DM axions might be contained within such bound structures [56,58]. Despite their low mass $\left(10^{-19} M_{\odot} \lesssim M_{\mathrm{AMC}} \lesssim 10^{-5} M_{\odot}\right)$ and large radius $\left(10^{-8} \lesssim R \lesssim 10^{-2} \mathrm{pc}\right)$, the density of an AMC can be many orders of magnitude larger than the local DM density [59]. On the other hand, AMCs are significantly more diffuse than stars. In our companion paper [60], we show that tidal interactions with stars can have a dramatic effect on the survival of AMCs in the Milky Way (MW). AMCs toward the inner regions of the Galaxy undergo significant 
stripping and disruption, whereas those further out remain intact. This, in turn, is reflected in the observational signatures of AMCs.

Here, we build upon the results of [60] to predict the rate, brightness, and sky distributions of encounters between AMCs and NSs. For a typical MW virial velocity of $v \sim 200 \mathrm{~km} / \mathrm{s} \sim 10^{-11} \mathrm{pc} / \mathrm{s}$, the time taken for a NS to pass through an $\mathrm{AMC}$ is expected to be $\mathcal{O}\left(10^{3}-10^{9}\right) \mathrm{s}$, meaning that these interactions would appear as radio "transients." As we will show, this is particularly true for the brightest events, which last between $10^{5}$ and $10^{7} \mathrm{~s}$. We consider a KimShifman-Vainshtein-Zakharov-like QCD axion $[61,62]$ of mass $m_{a}=20 \mu \mathrm{eV}$, motivated by recent simulations $[58,63]$. This corresponds to a radio frequency of $f=4.84 \mathrm{GHz}$ and an axion-photon coupling of $g_{\text {ary }} \approx 8 \times 10^{-15} \mathrm{GeV}^{-1}$. All code associated with this Letter is available online [64] and makes use of the Python scientific computing packages NumPy [65] and SciPy [66], as well as the graphics environment Matplotlib [67].

Axion miniclusters in the Milky Way.-Because of the randomness of the initial overdensity fluctuations, miniclusters are born with a wide range of masses and densities. During matter domination, the minicluster halo mass function (HMF) evolves under hierarchical structure formation, allowing ever heavier AMCs to form. $N$-body simulations modeling AMC evolution from recombination to $z \approx 99$ predict a featureless HMF with a characteristic slope $d n / d \log M \sim M^{-0.7}$ [33], corroborating semianalytic studies $[36,68,69]$. We consider AMC masses between $3.3 \times 10^{-19} \leq M_{\mathrm{AMC}} / M_{\odot} \leq 5.1 \times 10^{-5}[68,69]$ and assume that these AMCs make up 100\% of the DM, tracing the Navarro-Frenk-White (NFW) profile of the MW halo. Miniclusters are also characterized by their overdensity parameter $\delta$, which depends upon the random initial conditions of the axion field and its gradient at the onset of axion oscillations [29-31]. We take the distribution of $\delta$ from recent simulations [58] and map it to the AMC characteristic density $\rho_{\text {AMC }}$ as in Ref. [31].

Tidal interactions between AMCs and their local environment can have a significant effect on AMC properties [60,70-73]. Stellar encounters prove to be the most important and can easily lead to the total disruption of AMCs. In addition, many successive weak encounters can cause surviving AMCs to lose mass, as well as altering their internal density. In [60], we present Monte Carlo simulations used to assess the effects of these stellar tidal interactions, starting from the initial distribution of masses and densities described above. These simulations allow us to describe the properties of AMCs across the MW today.

The internal density profiles of AMCs are not well understood. For example, Ref. [33] finds that AMCs with masses $M_{\mathrm{AMC}} \gtrsim 10^{-13} M_{\odot}$ have approximately NFW [74] profiles in their outer regions, whereas lighter AMCs are expected to have power-law (PL) profiles [57]. For each simulation, we therefore treat the entire population of AMCs as having a single universal structure given by either a NFW or PL density profile (see Supplemental Material [35] for examples). For a fixed characteristic density $\rho_{\text {AMC }}$, the mean internal density $\bar{\rho}$ of our assumed NFW profile is $\mathcal{O}\left(10^{5}\right)$ times lower than for the PL profile. This leads to quantitative differences in the survival probability and distributions of masses and radii. Using these two density profiles therefore allows us to generously bound the uncertainties coming from the internal AMC structure.

Another source of uncertainty is related to the formation of axion stars (ASs). These are nonrelativistic compact objects, described by solutions to the Schrödinger-Poisson equation $[29,75]$, which can potentially form in the centers of miniclusters [76-78]. ASs have an inverse relationship between their mass and radius, leading to a potentially problematic scenario for a low-mass AMC in which its radius is smaller than that of the AS in its center. To avoid this issue, we first follow the evolution of all AMCs, described initially by the HMF above, then apply a cut to remove these potentially problematic light AMCs [79]. The remaining miniclusters form our fiducial sample and are used throughout the rest of this Letter (see Supplemental Material [35] and our companion paper [60] for further details).

Minicluster-neutron star encounters.-The AMC-NS encounter cross section is given by $\sigma(u)=\pi R^{2}[1+$ $\left.2 G M_{\mathrm{NS}} /\left(R u^{2}\right)\right]$, where $R$ is the minicluster radius and $M_{\mathrm{NS}}=1.4 M_{\odot}$ is the NS mass. This expression includes a gravitational focusing term [[80], p. 627] that depends on the relative velocity of the encounter $u$. We assume that the velocity dispersion of the NS and minicluster populations is $\sigma_{v}(r)=\sqrt{G M_{\text {encl }}(r) / r} \approx 200 \mathrm{~km} / \mathrm{s}$, depending on the enclosed MW mass $M_{\text {encl }}(r)$ within a galactocentric radius $r$. The encounter velocity then follows a Maxwell-Boltzmann distribution $f_{u}(u)$ with dispersion $\sigma_{u}(r)=\sqrt{2} \sigma_{v}(r)$ ([80] problem 8.8 ). The velocity-weighted cross section can then be written as

$\langle\sigma u\rangle(r) \equiv \int d^{3} u f_{u}(u) u \sigma(u) \approx \sqrt{8 \pi} \sigma_{u}(r) R^{2}(1+\hat{R} / R)$,

where $\hat{R}=G M_{\mathrm{NS}} / \sigma_{u}^{2} \sim \mathcal{O}\left(10^{-7} \mathrm{pc}\right)$.

Interactions on the outskirts of large, diffuse AMCs dominate the encounter rate but do not produce a significant increase in the axion density close to the NS. We therefore consider only interactions with impact parameters $b<b_{\text {cut }}$ (with $b_{\text {cut }} \leq R$ ), such that the peak overdensity during the encounter is at least $10 \%$ of the local DM density $\rho_{\mathrm{DM}}(r)$. We parametrize this in terms of an effective cross section $\langle\widetilde{\sigma u}\rangle(r)$, which saturates at the standard cross section $\langle\sigma u\rangle(r)$ for dense AMCs. The expected rate of encounters over the entire MW is then $[81,82]$

$$
\Gamma=\int d^{3} \mathbf{r} \int d R \frac{d n_{\mathrm{AMC}}(r)}{d R} n_{\mathrm{NS}}(\mathbf{r})\langle\widetilde{\sigma u}\rangle(r),
$$

where $n_{\mathrm{NS}}(\mathbf{r})$ is the NS number density at position $\mathbf{r}$ and $d n_{\mathrm{AMC}}(r) / d R$ is the differential number density of AMCs 


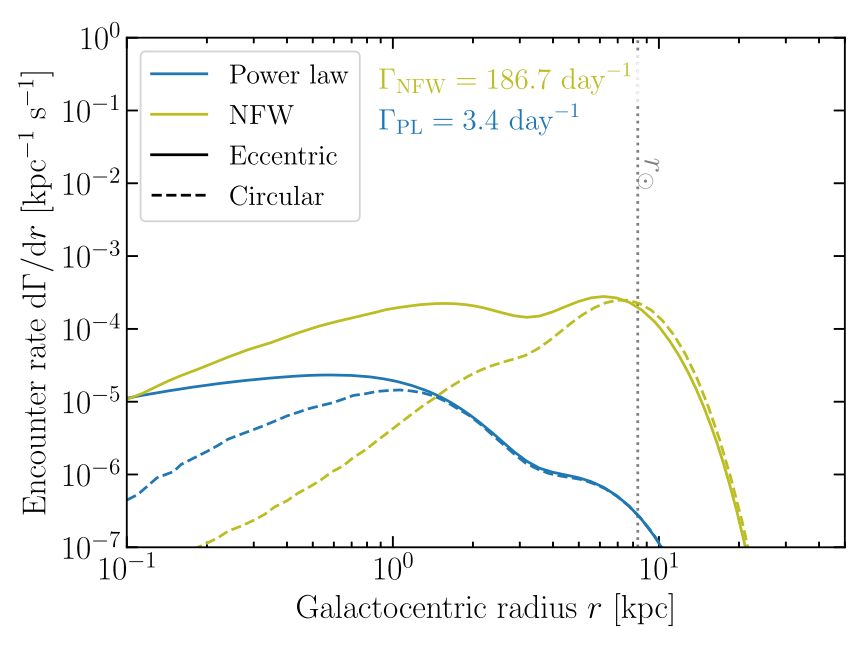

FIG. 1. AMC-NS encounter rate incorporating the effects of AMC disruptions due to stellar encounters. We show results assuming that AMCs are solely on circular orbits (dashed) and assuming a distribution of orbital eccentricities (dotted). The vertical dotted line marks the position of the Solar System, $r_{\odot}$.

with radius $R$, computed in [60]. Taking into account the distribution of AMC properties, we find that $d \Gamma / d M_{\mathrm{AMC}} \sim$ $1 / M_{\mathrm{AMC}}$, rising more steeply than this at low AMC masses, where the gravitational focusing effect becomes important for $R \lesssim \hat{R}$. We assume a population of $10^{9} \mathrm{NSs}$ in the MW, with $60 \%$ formed in the bulge and $40 \%$ in the disk $[83,84]$, of which $20 \%$ have become unbound due to natal kicks [84]. Explicit expressions for $n_{\mathrm{NS}}(\mathbf{r})$ are given in the Supplemental Material [35].

In Fig. 1, we show the integrand $d \Gamma / d r$ of Eq. (3). At the largest radii $(r \gtrsim 10-20 \mathrm{kpc})$, encounters are rare due to the falling number of both AMCs and NSs. Near the Galactic Center, the densities of both AMCs and NSs instead rise rapidly. However, the encounter rate is suppressed by the low survival probability of AMCs in this dense environment, leading to a plateau. Note that the dip at $3-4 \mathrm{kpc}$ is a coincidence between the falling survival probability toward the Galactic Center and scale at which the bulge population of NSs becomes dominant. We find that the encounter rate is larger for eccentric than for circular orbits; AMCs on eccentric orbits spend less time at small radii, leading to a larger survival probability. For NFW profiles, there is a comparable contribution from encounters with bulge NSs at small radii and encounters with disk NSs at larger radii (close to the solar circle $r_{\odot}$ ). Miniclusters with PL profiles are more dense and therefore smaller than those with NFW profiles, leading to an overall decrease in the encounter rate. However, these dense AMCs are also more resistant to disruption in the Galactic Center, leading to a greater survival probability at small $r$. This compensates for their smaller size and means that for PL miniclusters most encounters occur with NSs in the bulge. Over the entire Galaxy, we expect encounter rates of $\Gamma_{\mathrm{PL}}=3.4$ and
$\Gamma_{\mathrm{NFW}}=186.7 \mathrm{day}^{-1}$. If we had neglected the stellar disruption of AMCs described in [60], these encounter rates would be larger by a factor of 1.4 and 45.4 for PL and NFW profiles, respectively.

Signal estimation.-For each choice of minicluster profile, we sample $10^{7}$ encounters to calculate the expected distributions of fluxes, durations, and sky locations. We sample the galactocentric radius of the encounter according to the encounter rate $d \Gamma / d r$ in Fig. 1 . We draw the height of the encounter $z_{\text {cyl }}$ above the Galactic plane from the distribution of NSs along the $z_{\mathrm{cyl}}$ axis (assuming that the AMC distribution is spherically symmetric), and we draw the galactocentric azimuth angle uniformly between 0 and $2 \pi$. We sample the AMC radius $R$ following $d \Gamma / d R$ at fixed galactocentric radius and sample the AMC density, given $R$, from the distributions derived in [60]. The impact parameter $b \in\left[0, b_{\text {cut }}\right]$ is sampled according to $P(b) \propto b$.

The NS magnetic field at the poles $B_{0}$ and the period $P$ are drawn from log-normal distributions, with mean and dispersion given by $\log _{10}(B / \mathrm{G})=12.65, \sigma_{B}=0.55[85,86]$, and $\log _{10}(P / \mathrm{ms})=2.7, \sigma_{P}=-0.34$ [87] respectively.

Considering the trajectories of individual axions close to the NS, the maximum impact parameter that still crosses the conversion radius $R_{c}$ is

$$
b_{\max }=R_{c} \sqrt{1+\frac{2 G M_{\mathrm{NS}}}{u^{2} R_{c}}} \sim \mathcal{O}\left(10^{-9}\right) \mathrm{pc} .
$$

AMCs have radii several orders of magnitude larger than this, so we can consider the NS as tracing the internal AMC density $\rho_{\text {int }}(R)$ during the encounter. Such a direct encounter is likely to completely disrupt the AMC. However, the relaxation time for the $\mathrm{AMC}$ is much longer than the encounter time and we therefore neglect the evolution of the minicluster during the NS transit.

For each encounter, we estimate the radio flux density

$$
\mathcal{S}=\frac{1}{\mathrm{BW}} \frac{1}{4 \pi s^{2}} \frac{d \mathcal{P}_{a}}{d \Omega},
$$

where $s$ is the distance of the encounter from Earth. The signal bandwidth (BW) is typically set by the axion velocity dispersion far from the NS [23], leading to narrow band line emission. However, because of the small internal velocity dispersion of the AMCs $(\lesssim 1 \mathrm{~km} / \mathrm{s})$, this is unlikely to be the main source of the signal bandwidth. We therefore fix the bandwidth of the signal to a larger value, $1 \mathrm{kHz}$, representative of the resolution of current and planned radio telescopes [88-90]. Determining the full directional dependence of the radio emission is highly nontrivial, though there have been a number of recent developments dealing, for example, with nonradial infall of axions $[26,91]$. Here, we assume for simplicity that the emission is isotropic; Eq. (1) has been averaged over viewing angle and we have fixed $R_{c}$ to its angular average. If future studies show that the radio emission is instead 

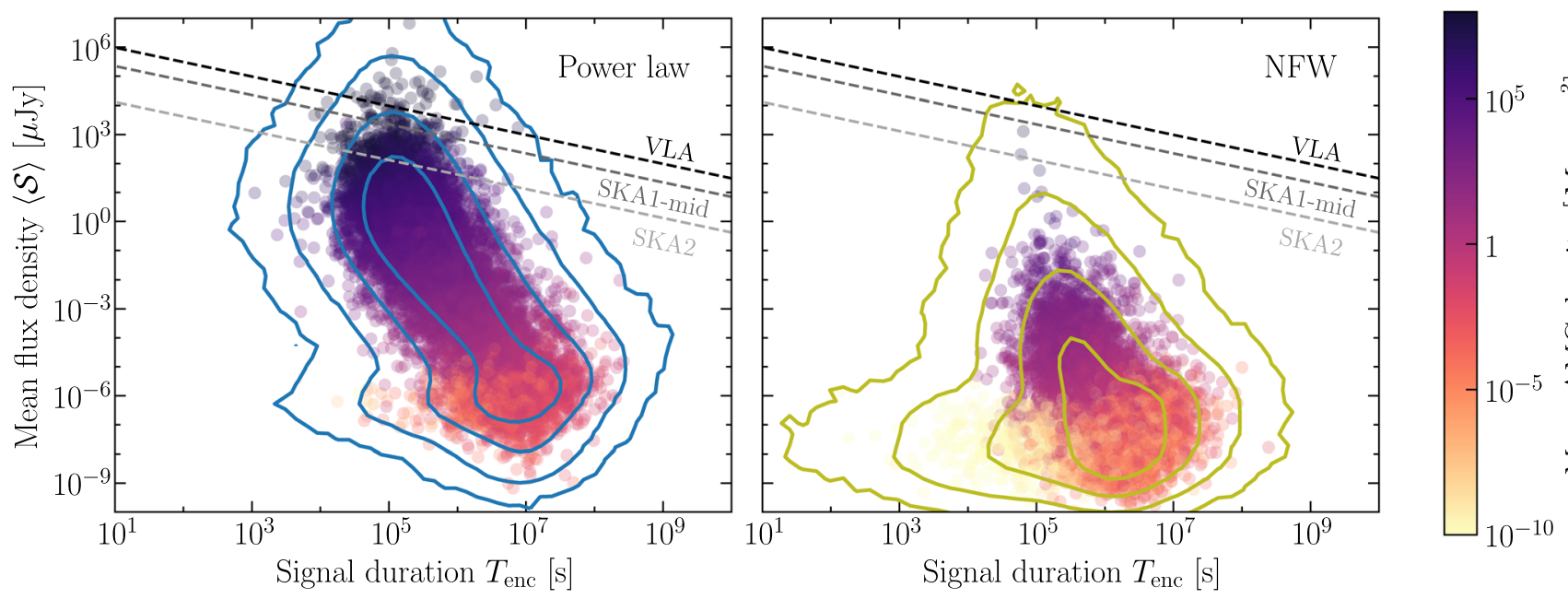

FIG. 2. Mean flux density and duration of the radio signal for a random sample of $10^{4}$ AMC-NS encounters, colored by mean AMC density. Overlaid are 1-, 2-, 3-, and 4- $\sigma$ contours for the distribution of $\langle\mathcal{S}\rangle$ and $T_{\text {enc }}$, derived from a larger sample of $10^{7}$ simulations. The diagonal dashed lines show the minimum $5 \sigma$ detectable flux estimated using the radiometer equation [92] (where we assume that the observation time equals the duration of the encounter) for the VLA [88], SKA1-mid [93,94], and SKA2 [24]. We assume AMCs have power-law (left) or NFW (right) internal density profiles.

concentrated in a fraction $f_{\text {beam }}$ of the unit sphere, then our results can be straightforwardly reinterpreted: the observed rate will be reduced by a factor $\sim f_{\text {beam }}$ and the flux density increased by a corresponding factor $\sim f_{\text {beam }}$.

We estimate the mean flux density $\langle\mathcal{S}\rangle$ of each encounter by averaging Eq. (5) over the duration of the encounter $T_{\text {enc }}=2 \sqrt{R^{2}-b^{2}} / u$. Note that the peak flux during the encounter is generally comparable to the mean flux. Since each encounter is independent, the number of expected encounters starting within a time step $\Delta t$ is Poisson distributed with mean $\Gamma \Delta t$. Our simulations can therefore be combined into a time series for the predicted signal by taking a uniform distribution of start times. In Fig. 2, we show the distributions of $T_{\text {enc }}$ and $\langle\mathcal{S}\rangle$ derived from our full sample of AMC-NS encounters. We also plot a smaller sample of $10^{4}$ individual encounters, colored by the mean internal density of the AMC.

For PL AMCs, the distribution of encounters peaks at flux densities between $10^{-6}$ and $10^{2} \mu \mathrm{Jy}$, with a typical duration of 1-100 days. The distribution also includes a number of bright events $\mathcal{O}(1 \mathrm{Jy})$, which should be detectable by current radio telescopes such as the Very Large Array (VLA) [95]. These brightest events come from encounters with dense AMCs $\left(\gtrsim 10^{5} M_{\odot} \mathrm{pc}^{-3}\right)$. AMCs with NFW internal density profiles have a density around $10^{5}$ times smaller than their PL counterparts, making highflux events rarer. However, this is partially compensated by the larger encounter rate between NSs and NFW AMCs. The rate of encounters above a flux of 1 mJy (a sensitivity which has been achieved in recent searches for NS radio emission $[26,27,96])$ is $\Gamma_{\mathrm{PL}}(\Psi>1 \mathrm{mJy})=0.04 /$ day and $\Gamma_{\mathrm{NFW}}(\Psi>1 \mathrm{mJy})=0.007 /$ day for PL and NFW miniclusters, respectively. Given the rate and duration of the encounters, we expect at least one bright event in the sky at all times. We also note that the rate of the brightest events $(\langle\mathcal{S}\rangle>1 \mathrm{Jy})$ is relatively insensitive to our assumptions on the AMC density profile, once stellar perturbations are taken into account (see Supplemental Material [35]).

The sky distribution of AMC-NS encounters is shown in Fig. 3 for AMCs with NFW profiles. In this case, the encounters occur predominantly toward the Galactic Center, although there is also a population of events extending along the disk, to a longitude of $|\ell| \lesssim 60^{\circ}$. This morphology reflects the two populations of NSs in the bulge and disk. In the case of PL AMCs (not shown), encounters are concentrated almost exclusively toward the

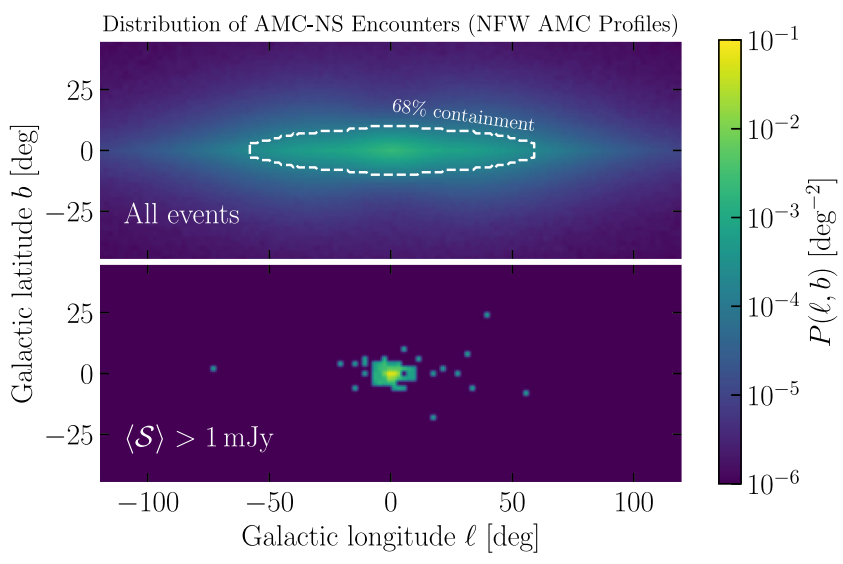

FIG. 3. Expected distribution of all AMC-NS encounters (top) and bright AMC-NS encounters (bottom), assuming NFW internal AMC density profiles. For AMCs with PL density profiles, $68 \%$ of all encounters lie within $7^{\circ}$ of the Galactic Center. 
Galactic Center, with $68 \%$ of events lying within $7^{\circ}$ of the center. Considering only the brightest events, we find that the distributions become even more concentrated toward the Galactic Center, as shown in the bottom panel of Fig. 3.

Discussion and conclusion.-In this Letter, we have characterized the radio signatures of axion-photon conversion from encounters between NSs in the Milky Way and a population of QCD axion miniclusters (see companion paper [60]). These signatures will appear as regular transient radio point sources (Fig. 1) with timescales varying from days to over a year. Interestingly, these transients will be spatially clustered toward the Galactic Center (Fig. 3) with potentially observable fluxes (Fig. 2). This suggests that radio observations could be used to discover QCD axion DM in the near future.

Within the MW, there are a variety of sources of transient radio emission, especially from the Galactic Center. A recent analysis of archival VLA data [97] found a number of potential transients within $\sim 0.001^{\circ}$ of $\mathrm{Sgr} \mathrm{A}^{*}$ (see also Ref. [98]). Potential explanations of these radio transients are pulsar emission, radio flares from dwarf stars, and outflows from x-ray binaries, all of which emit a broad energy spectrum. AMC-NS encounters could contribute to a population of transients toward the Galactic Center. However, our results predict a characteristic linelike emission that would need to be confirmed with dedicated search strategies.

The slope of the AMC halo mass function is not well constrained $[33,36,68,69]$. To test the dependence of our results on this slope, we reran the entire pipeline assuming $d n / d \log M \sim M^{-0.5}$, as obtained using the Press-Schechter formalism [68]. Flattening the HMF increases the mean AMC mass and therefore reduces the total number of AMCs in the MW. (Note that the brightness of an individual event is only mildly dependent on the AMC mass.) Fortunately, this is partially counteracted by an increase in the encounter cross section in Eq. (2), which scales as $R^{2} \sim M^{2 / 3}$. Overall, we find that the encounter rate has only a mild dependence on the slope; flattening from -0.7 to -0.5 leads to a factor of 5-10 fewer events above $1 \mathrm{mJy}$. A recent study [99] found an HMF slope that broadly agrees with the results of Ref. [33], but with an overall shift to lower masses. This would primarily result in a decrease to the number of AMCs passing the AS cut and therefore a reduced encounter rate.

The typical AMC density $\rho_{\mathrm{AMC}} \sim \delta^{4}$ is more strongly affected by the uncertainty in the AMC internal density profile $\left(\bar{\rho}_{\mathrm{PL}} / \bar{\rho}_{\mathrm{NFW}} \sim 10^{5}\right)$ than by a change in $\delta$. We therefore do not expect that small variations in the distribution of $\delta$ would affect the detectability of the signal.

The production of axion miniclusters in the early Universe is a robust prediction of the postinflationary scenario of axion cosmology [28-32]. The fraction of axions bound in these structures remains unclear [33], but is likely to be substantial. As we show in [60], if this fraction is large then direct detection efforts may be ineffective. Our results are therefore complementary to these ongoing direct searches, alongside searches for continuous radio emission from the smooth halo of axions interacting with NSs [21-24].

Although we have calculated the population-level distribution of signals, much work is still needed to characterize the details of each event. More concretely, the precise signal bandwidth [100] and the modeling of the conversion process in realistic NS magnetospheres remain unclear. Both of these can have dramatic effects on the properties of the final signal and should be addressed in future work. We therefore emphasize that a nondetection cannot be reliably used to set upper limits on the axion parameter space. Nevertheless, this Letter characterizes the unique transient nature of these interactions and shows that current and near future radio telescopes have the sensitivity required to detect QCD axion DM.

Supporting data for this Letter are openly available from the Zenodo repository [64].

We thank Sebastian Baum, Gianfranco Bertone, Malte Buschmann, Matthew Lawson, David J.E. Marsh, M. C. David Marsh, Alexander Millar, Lina Necib, Ciaran O'Hare, Javier Redondo, and Ben Safdi for providing insightful comments. T.E. acknowledges support by the Vetenskapsrådet (Swedish Research Council) through Contract No. 638-2013-8993 and the Oskar Klein Centre for Cosmoparticle Physics. T. E. was also supported in part by the research environment grant "Detecting Axion Dark Matter in the Sky and in the Lab (AxionDM)" funded by the Swedish Research Council (VR) under Dnr 2019-02337. T. E. and C. W. are supported by the NWO through the VIDI research program "Probing the Genesis of Dark Matter" (680-47-5). L. V. is supported through the research program "The Hidden Universe of Weakly Interacting Particles" with Project No. 680.92.18.03 (NWO Vrije Programma), which is partly financed by the Nederlandse Organisatie voor Wetenschappelijk Onderzoek (Dutch Research Council), and acknowledges support from the European Union's Horizon 2020 Research and Innovation Programme under the Marie Skłodowska-Curie Grant Agreement No. 754496 (H2020-MSCA-COFUND-2016 FELLINI). B. J. K. thanks the Spanish Agencia Estatal de Investigación (AEI, MICIU) for the support to the Unidad de Excelencia María de Maeztu Instituto de Física de Cantabria, ref. MDM-2017-0765. Some of this work was carried out on the Dutch national e-infrastructure with the support of SURF Cooperative.

*thomas.edwards@fysik.su.se

†kavanagh@ifca.unican.es

"luca.visinelli@sjtu.edu.cn

§c.weniger@uva.nl

[1] R. D. Peccei and H. R. Quinn, Phys. Rev. Lett. 38, 1440 (1977). 
[2] R. D. Peccei and H. R. Quinn, Phys. Rev. D 16, 1791 (1977).

[3] S. Weinberg, Phys. Rev. Lett. 40, 223 (1978).

[4] F. Wilczek, Phys. Rev. Lett. 40, 279 (1978).

[5] A. Vilenkin, Phys. Rev. D 24, 2082 (1981).

[6] L. F. Abbott and P. Sikivie, Phys. Lett. 120B, 133 (1983).

[7] M. Dine and W. Fischler, Phys. Lett. 120B, 137 (1983).

[8] J. Preskill, M. B. Wise, and F. Wilczek, Phys. Lett. 120B, 127 (1983).

[9] Y. Nambu, Phys. Rev. 117, 648 (1960).

[10] J. Goldstone, Nuovo Cimento 19, 154 (1961).

[11] J. Goldstone, A. Salam, and S. Weinberg, Phys. Rev. 127, 965 (1962).

[12] P. Sikivie, Phys. Rev. Lett. 51, 1415 (1983); 52, 695(E) (1984).

[13] P. Sikivie, Phys. Rev. D 32, 2988 (1985); 36, 974(E) (1987).

[14] I. G. Irastorza and J. Redondo, Prog. Part. Nucl. Phys. 102, 89 (2018).

[15] P. Sikivie, Rev. Mod. Phys. 93, 015004 (2021).

[16] D. Cadamuro, Cosmological limits on axions and axionlike particles, Ph.D. thesis, Munich U., arXiv:1210.3196.

[17] A. Ayala, I. Domínguez, M. Giannotti, A. Mirizzi, and O. Straniero, Phys. Rev. Lett. 113, 191302 (2014).

[18] N. Vinyoles, A. Serenelli, F. L. Villante, S. Basu, J. Redondo, and J. Isern, J. Cosmol. Astropart. Phys. 10 (2015) 015.

[19] J. S. Lee, arXiv:1808.10136.

[20] F. Calore, P. Carenza, M. Giannotti, J. Jaeckel, and A. Mirizzi, Phys. Rev. D 102, 123005 (2020).

[21] M. S. Pshirkov and S. B. Popov, J. Exp. Theor. Phys. 108, 384 (2009).

[22] F. P. Huang, K. Kadota, T. Sekiguchi, and H. Tashiro, Phys. Rev. D 97, 123001 (2018).

[23] A. Hook, Y. Kahn, B. R. Safdi, and Z. Sun, Phys. Rev. Lett. 121, 241102 (2018).

[24] B. R. Safdi, Z. Sun, and A. Y. Chen, Phys. Rev. D 99, 123021 (2019).

[25] T. D. P. Edwards, M. Chianese, B. J. Kavanagh, S. M. Nissanke, and C. Weniger, Phys. Rev. Lett. 124, 161101 (2020).

[26] J. W. Foster, Y. Kahn, O. Macias, Z. Sun, R. P. Eatough, V. I. Kondratiev, W. M. Peters, C. Weniger, and B. R. Safdi, Phys. Rev. Lett. 125, 171301 (2020).

[27] J. Darling, Astrophys. J. Lett. 900, L28 (2020).

[28] C. J. Hogan and M. J. Rees, Phys. Lett. B 205, 228 (1988).

[29] E. W. Kolb and I. I. Tkachev, Phys. Rev. Lett. 71, 3051 (1993).

[30] E. W. Kolb and I. I. Tkachev, Phys. Rev. D 49, 5040 (1994).

[31] E. W. Kolb and I. I. Tkachev, Phys. Rev. D 50, 769 (1994).

[32] E. W. Kolb and I. I. Tkachev, Astrophys. J. Lett. 460, L25 (1996).

[33] B. Eggemeier, J. Redondo, K. Dolag, J. C. Niemeyer, and A. Vaquero, Phys. Rev. Lett. 125, 041301 (2020).

[34] P. Goldreich and W. H. Julian, Astrophys. J. 157, 869 (1969).

[35] SeeSupplemental Material athttp://link.aps.org/supplemental/ 10.1103/PhysRevLett.127.131103 for a discussion of neutron star populations and magnetosphere modeling; a detailed description of the axion minicluster density profiles; details of the flux distributions; and a discussion of the role of axion stars, which includes Refs. [36-54].

[36] D. Ellis, D. J. E. Marsh, and C. Behrens, Phys. Rev. D 103, 083525 (2021).

[37] C. A. J. O'Hare and A. M. Green, Phys. Rev. D 95, 063017 (2017).

[38] I. I. Tkachev, Phys. Lett. B 261, 289 (1991).

[39] D. J. Kaup, Phys. Rev. 172, 1331 (1968).

[40] R. Ruffini and S. Bonazzola, Phys. Rev. 187, 1767 (1969).

[41] M. Colpi, S. L. Shapiro, and I. Wasserman, Phys. Rev. Lett. 57, 2485 (1986).

[42] S. Raby, Phys. Rev. D 94, 103004 (2016).

[43] T. Dietrich, F. Day, K. Clough, M. Coughlin, and J. Niemeyer, Mon. Not. R. Astron. Soc. 483, 908 (2019).

[44] L. Visinelli, S. Baum, J. Redondo, K. Freese, and F. Wilczek, Phys. Lett. B 777, 64 (2018).

[45] H.-Y. Schive, M.-H. Liao, T.-P. Woo, S.-K. Wong, T. Chiueh, T. Broadhurst, and W. Y. Pauchy Hwang, Phys. Rev. Lett. 113, 261302 (2014).

[46] R. N. Manchester, G. B. Hobbs, A. Teoh, and M. Hobbs, Astron. J. 129, 1993 (2005).

[47] J. Binney, O. Gerhard, and D. Spergel, Mon. Not. R. Astron. Soc. 288, 365 (1997).

[48] N. Bissantz and O. Gerhard, Mon. Not. R. Astron. Soc. 330, 591 (2002).

[49] P. J. McMillan, Mon. Not. R. Astron. Soc. 414, 2446 (2011).

[50] R. T. Bartels, T. D. P. Edwards, and C. Weniger, Mon. Not. R. Astron. Soc. 481, 3966 (2018).

[51] P. Haensel, V. A. Urpin, and D. G. Iakovlev, Astron. Astrophys. 229, 133 (1990), https://ui.adsabs.harvard .edu/abs/1990A\%26A...229..133H/abstract.

[52] P. Goldreich and A. Reisenegger, Astrophys. J. 395, 250 (1992).

[53] D. A. Shalybkov and V. A. Urpin, Mon. Not. R. Astron. Soc. 273, 643 (1995).

[54] J. A. Pons and U. Geppert, Astron. Astrophys. 470, 303 (2007).

[55] E. S. Phinney and S. R. Kulkarni, Annu. Rev. Astron. Astrophys. 32, 591 (1994).

[56] A. Vaquero, J. Redondo, and J. Stadler, J. Cosmol. Astropart. Phys. 04 (2019) 012.

[57] K. M. Zurek, C. J. Hogan, and T. R. Quinn, Phys. Rev. D 75, 043511 (2007).

[58] M. Buschmann, J. W. Foster, and B. R. Safdi, Phys. Rev. Lett. 124, 161103 (2020).

[59] L. Visinelli and J. Redondo, Phys. Rev. D 101, 023008 (2020).

[60] B. J. Kavanagh, T. D. P. Edwards, L. Visinelli, and C. Weniger, companion paper, Phys. Rev. D 104, 063038 (2021).

[61] J. E. Kim, Phys. Rev. Lett. 43, 103 (1979).

[62] M. A. Shifman, A. I. Vainshtein, and V. I. Zakharov, Nucl. Phys. B166, 493 (1980).

[63] V. B. Klaer and G. D. Moore, J. Cosmol. Astropart. Phys. 11 (2017) 049. 
[64] B. J. Kavanagh, T. D. P. Edwards, and L. Visinelli, axionminiclusters [computer software], Zenodo, github.com/ bradkav/axion-miniclusters, https://doi.org/10.5281/zenodo .4006128 (2020).

[65] T. E. Oliphant, NumPy: A guide to NumPy (CreateSpace Independent Publishing Platform, North Charleston, 2015).

[66] P. Virtanen, R. Gommers, T. E. Oliphant, M. Haberland, T. Reddy et al., Nat. Methods 17, 261 (2020).

[67] J. D. Hunter, Comput. Sci. Eng. 9, 90 (2007).

[68] M. Fairbairn, D. J. E. Marsh, J. Quevillon, and S. Rozier, Phys. Rev. D 97, 083502 (2018).

[69] M. Fairbairn, D. J. E. Marsh, and J. Quevillon, Phys. Rev. Lett. 119, 021101 (2017).

[70] H.-S. Zhao, J. Taylor, J. Silk, and D. Hooper, arXiv: astro-ph/0502049.

[71] P. Tinyakov, I. Tkachev, and K. Zioutas, J. Cosmol. Astropart. Phys. 01 (2016) 035.

[72] V. S. Berezinsky, V. I. Dokuchaev, and Y. N. Eroshenko, J. Cosmol. Astropart. Phys. 11 (2013) 059.

[73] V. I. Dokuchaev, Y. N. Eroshenko, and I. I. Tkachev, J. Exp. Theor. Phys. 125, 434 (2017).

[74] J. F. Navarro, C. S. Frenk, and S. D. M. White, Astrophys. J. 462, 563 (1996).

[75] E. Seidel and W.-M. Suen, Phys. Rev. Lett. 72, 2516 (1994).

[76] D. G. Levkov, A. G. Panin, and I. I. Tkachev, Phys. Rev. Lett. 121, 151301 (2018).

[77] B. Eggemeier and J.C. Niemeyer, Phys. Rev. D 100, 063528 (2019).

[78] J. Chen, X. Du, E. W. Lentz, D. J. E. Marsh, and J. C. Niemeyer, arXiv:2011.01333.

[79] In our companion paper [60], we also include an initial mass loss of 5\%-40\% for AMCs with NFW profiles, due to tidal stripping from the DM halo of the MW.

[80] J. Binney and S. Tremaine, in Galactic Dynamics: Second Edition, edited by J. Binney and S. Tremaine (Princeton University Press, Princeton, NJ, 2008), ISBN 978-0-69113026-2 (HB).

[81] J. H. Buckley, P. S. Bhupal Dev, F. Ferrer, and F. P. Huang, Phys. Rev. D 103, 043015 (2021).
[82] A. Prabhu and N. M. Rapidis, J. Cosmol. Astropart. Phys. 10 (2020) 054.

[83] E. O. Ofek, Publ. Astron. Soc. Pac. 121, 814 (2009).

[84] N. Sartore, E. Ripamonti, A. Treves, and R. Turolla, Astron. Astrophys. 510, A23 (2010).

[85] C.-A. Faucher-Giguere and V. M. Kaspi, Astrophys. J. 643, 332 (2006).

[86] S. Bates, D. Lorimer, A. Rane, and J. Swiggum, Mon. Not. R. Astron. Soc. 439, 2893 (2014).

[87] D. R. Lorimer et al., Mon. Not. R. Astron. Soc. 372, 777 (2006).

[88] R. A. Perley, C. J. Chandler, B. J. Butler, and J. M. Wrobel, Astrophys. J. Lett. 739, L1 (2011).

[89] SKA Science Working Group, SKA1 system baseline design (2013), https://india.skatelescope.org/key-documents/.

[90] R. Braun, A. Bonaldi, T. Bourke, E. Keane, and J. Wagg, arXiv:1912.12699.

[91] M. Leroy, M. Chianese, T. D. P. Edwards, and C. Weniger, Phys. Rev. D 101, 123003 (2020).

[92] R. Dewey, G. Stokes, D. Segelstein, J. Taylor, and J. Weisberg, in Birth and Evolution of Neutron Stars: Issues Raised by Millisecond Pulsars, edited by S. P. Reynolds and D. R. Stinebring (National Radio Astronomy Observatory (NRAO), Green Bank, 1984), p. 234.

[93] F. Calore, M. Di Mauro, F. Donato, J. W. T. Hessels, and C. Weniger, Astrophys. J. 827, 143 (2016).

[94] D. Gaggero, G. Bertone, F. Calore, R. M. T. Connors, M. Lovell, S. Markoff, and E. Storm, Phys. Rev. Lett. 118, 241101 (2017).

[95] P. J. Napier, A. R. Thompson, and R. D. Ekers, IEEE Proc. 71, 1295 (1983).

[96] J. Darling, Phys. Rev. Lett. 125, 121103 (2020).

[97] A. Chiti, S. Chatterjee, R. Wharton, J. Cordes, T. J. W. Lazio, D. L. Kaplan, G. C. Bower, and S. Croft, Astrophys. J. 833, 11 (2016).

[98] J.-H. Zhao, M. R. Morris, and W. M. Goss, Astrophys. J. 905, 173 (2020).

[99] H. Xiao, I. Williams, and M. McQuinn, Phys. Rev. D 104, 023515 (2021).

[100] R. A. Battye, B. Garbrecht, J. I. McDonald, F. Pace, and S. Srinivasan, Phys. Rev. D 102, 023504 (2020). 